\title{
PHARYNGEAL-CERVICAL-BRACHIAL VARIANT: AN UNUSUAL PRESENTATION OF GUILLAIN-BARRÉ SYNDROME
}

\author{
Muhammad Furrukh', Saeed Bin Ayaz ${ }^{2}$ Fatima Ayaz ${ }^{1 \otimes}$
}

\begin{abstract}
Pharyngeal-cervical-brachial (PCB) variant is a rare presentation of Guillain-Barré syndrome (GBS), which is symbolized by oroparhyngeal, cervical and upper limb weakness. It is often wrongly diagnosed as brainstem stroke, myasthenia gravis or botulism. The presence of anti-GTIa and anti-GQIb ganglioside IgG antibodies and axonal electrophysiological pattern are the hallmark of PCB variant. The treatment is similar to any other variant of GBS. We present here, report of a 20 -year-old male, who presented with findings suggestive of PCB variant of GBS but had absent anti-GTIa and anti-GQIb antibodies and a normal cerebrospinal fluid. He made a remarkable recovery after administration of intravenous immunoglobulins.
\end{abstract}

KEY WORDS: Guillain-Barré syndrome (MeSH); Pharyngeal-cervical-brachial variant (Non-MeSH); anti-ganglioside antibodies (Non-MeSH); Immunoglobulin G (MeSH); Cerebrospinal Fluid (MeSH); Gangliosides (MeSH); GQIb ganglioside (MeSH); trisialoganglioside GTI (MeSH); Immunoglobulins (MeSH); Immunoglobulins, Intravenous (MeSH); Electrophysiology (MeSH).

THIS ARTICLE MAY BE CITED AS: Furrukh M, Ayaz SB, Ayaz F. Pharyngeal-cervical-brachial variant: an unusual presentation of Guillain-Barré syndrome. Khyber Med Univ J 2019;II(I):45-7 DOI: 10.35845/kmuj.2019.1827I.

\section{INTRODUCTION}

G uillain-Barré syndrome (GBS) is the most common cause of flaccid paralysis of acute onset worldwide.' The pharyngeal-cervical-brachial (PCB) presentation of GBS, is a rare variant that has an incidence rate of 0.07 $0.25 / 100,000 .^{2}$ It is described by weakness of facial muscles and upper limbs, dysarthria and loss of muscle stretch reflexes. ${ }^{3}$ The triggers for GBS e.g. infection, surgery and inflammation are also shared by its variants, particularly the PCB variant. ${ }^{3}$ The electro-diagnostic (EDX) evaluation of the $\mathrm{PCB}$ variant generally demonstrates an axonal pattern of polyneuropathy rather than demyelination. ${ }^{3}$ As this presentation of GBS is quite rare, the physicians often wrongly diagnose such patients as suffering from a brainstem stroke, botulism or myasthenia gravis. ${ }^{3}$ Anti-GTIa and anti-GQIb antibodies provide supportive evidence for the diagnosis of $\mathrm{PCB}$ variant though the former are positive in only $50 \%$ of the patients. ${ }^{3}$ The early disease may also lack the characteristic cerebrospinal fluid (CSF) or EDX abnormalities. ${ }^{3}$ We present here, report of a 20 -year-old male, who presented with findings suggestive of the $P C B$ variant of $G B S$ but had absent anti-GTIa and anti-GQIb antibodies and a normal CSF and EDX evaluation initially. He made a remarkable recovery after administration of intravenous immunoglobulins (IVIG).

\section{CASE SUMMARY}

A 20-year-old male presented to us with complaints of fever and productive cough for one month and inability to swallow, diplopia and nasal twang in voice for the last two days. Fever was insidious in onset, documented up to $100^{\circ} \mathrm{F}$, and intermittent in character with no diurnal variation. He was unable to
I Registrar Internal Medicine, Holy Family Hospital, Rawalpindi, Pakistan

2 Consultant, Department of Physical Medicine and Rehabilitation, Combined Military Hospital Quetta and Quetta Institute of Medical Sciences, Quetta, Pakistan

Email区 : fatimaqureshi56@yahoo.com Date Submitted: March 16,2018

Date Revised: $\quad$ March 28, 2019

Date Accepted: $\quad$ March 28, 2019

swallow both liquid and solid diet and had episodes of nasal regurgitation of food particles. The diplopia was evident on lateral gaze. He also had complaints of progressive weakness involving upper limbs more than lower limbs and difficulty in combing hairs, brushing teeth and writing. There was no history of loose stools.

On examination, he had a blood pressure of $150 / 100 \mathrm{mmHg}$ with a heart rate of 106 beats/min. He was maintaining oxygen saturation at air without oxygen support. The neurological examination revealed bilateral $6^{\text {th }}, 9^{\text {th }}, 10^{\text {th }}, 11^{\text {th }}$, and $12^{\text {th }}$ nerves' palsies. The muscle power was $3 / 5$ in upper limbs and $4 / 5$ in lower limbs according to the Medical Research Council Scale. ${ }^{4}$ The muscle stretch reflexes were grade 0 in upper limbs and $\mathrm{I}+$ in lower limbs with absent Babinski reflex. The sensations were intact. He had an ataxic gait; however, the cerebellar signs were absent. Based on history and clinical examination, a provisional diagnosis of $\mathrm{PCB}$ variant of GBS was made while keeping brain stem stroke, botulism and myasthenia gravis as differentials.

The laboratory evaluation revealed hemoglobin of $17 \mathrm{~g} / \mathrm{dl}$ and hematocrit of 45\%. The CSF evaluation revealed a normal study. Arterial blood gases and the pulmonary function tests were also normal. Rest of the baseline investigations were within normal limits. The magnetic resonance imaging (MRI) of the brain, cervical spine and chest $x$-rays did not show any abnormality. The EDX evaluation, performed on the third day of admission revealed a normal study. His antiganglioside antibodies' profile was negative. 

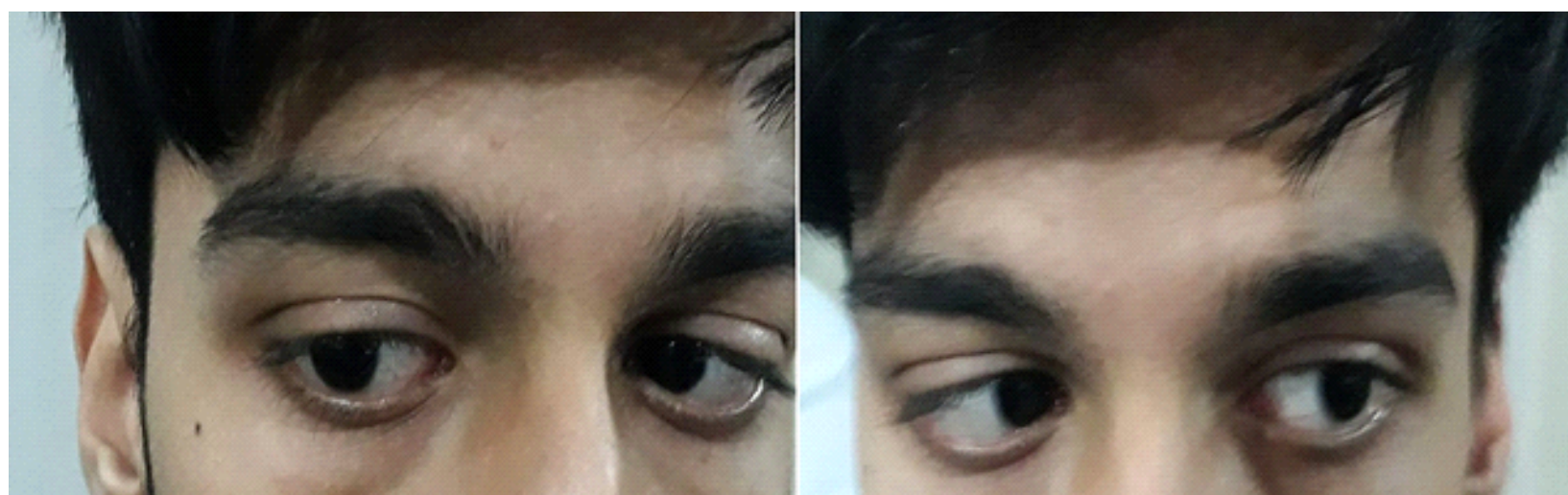

Figure I: Inability of patient to abduct eyes

Based on progressive and persistent weakness, absent muscle stretch reflexes and normal MRI brain and CSF, the patient was treated as a case of $\mathrm{PCB}$ variant of GBS. He was managed in the intensive care unit of the hospital. The feeding was started through a nasogastric tube. He received $125 \mathrm{~g}$ of IV IG over five days. His symptoms started improving on $6^{\text {th }}$ day of the start of treatment and the nasogastric tube was removed. An EDX assessment was recalled that suggested acute motor axonal neuropathy. The second CSF evaluation revealed a high protein content with a normal white blood cell count (albuminocytological dissociation). His follow-up examination after one week showed persistent inability to abduct eyes (Figure-I) while rest of the weakness was fully resolved. He was further advised wide range of motion exercises of the eyeballs. Written informed consent was obtained from the patient for publication of his personal information.

\section{DISCUSSION}

The first description of the PCB variant of GBS dates to 1986 when Ropper identified it as an atypical presentation of GBS in three patients who exhibited rapidly progressive weakness of the muscles of oral cavity, pharynx, neck and shoulder. The legs were spared and there were no sensory symptoms. ${ }^{5}$

The above-mentioned clinical features are still the hallmark of the PCB variant. History of a recent infection strongly supports the diagnosis. Investigations that validate the disease include albuminocytological dissociation of CSF, antibodies against GTIa or GQIb and neuropathy on EDX studies. ${ }^{3}$ Albuminocytological dissociation of CSF may possibly be absent in $50 \%$ of the patients during first week of the illness. ${ }^{3}$ Anti-GTIa antibodies are also nonexistent in half of the patients. ${ }^{6}$ The EDX studies in PCB reveal an axonal pattern of the polyneuropathy rather than demyelination. MRI brain and cervical spine are normal in the $\mathrm{PCB}$ variant.

The management guidelines for the $P C B$ variant are analogous to other variants of GBS. ${ }^{3}$ The do's and don'ts of IVIG or plasma exchange therapy are similar. ${ }^{3}$ In early disease, death may occur due to sepsis, pulmonary embolism or cardiac arrest so all patients should remain admitted in the hospital until no clinical progression of the morbidity is established.' Monitoring of bulbar function and respiratory effort is obligatory and directs the corresponding use of nasogastric tube and/or ventilator support. ${ }^{3}$ Ventilatory support may be required in patients with inefficient cough, atelectasis, hypoxemia, hypercarbia or vital capacity $<15 \mathrm{ml} / \mathrm{kg}$ body weight.

There has been a report of the PCB variant of GBS from Pakistan. ${ }^{7} \mathrm{He}$ was a 25-year-old male soldier with similar symptoms, normal CSF and axonal neuropathy on EDX evaluation. He remained on mechanical ventilation for 170 days. He received plasmapheresis and had a sub-total recovery after seven months. Our case had a milder course and a rapid recovery probably because of good initial motor power, early treatment and no requirement for the ventilatory support.

In conclusion, recognition of the $\mathrm{PCB}$ variant and other atypical cases of GBS is important, as it permits early, effective and lifesaving treatment in patients with a potential of highly morbid or fatal outcome.

\section{REFERENCES}

I. Yuki N, Hartung HP. Guillain-Barré syndrome. N Engl J Med 2012 366(24):2294-304. DOI: I0.1056/ NEJMral I 14525.

2. Emilia-Romagna Study Group on Clinical and Epidemiological Problems in Neurology. GuillainBarré syndrome variants in EmiliaRomagna, Italy, 1992-3: incidence, clinical features, and prognosis. J Neurol Neurosurg Psychiatry 1998;65(2):2।8-24. DOI: I0.1 |36/ jnnp.65.2.218.

3. Wakerley BR, Yuki N. Pharyngealcervical-brachial variant of GuillainBarré syndrome. J Neurol Neurosurg Psychiatry 2014;85(3): 339-44. DOI: $10.1136 /$ jnnp-2013 305397.

4. Compston A. Aids to the investigation of peripheral nerve injuries. Medical Research Council: Nerve Injuries Research Committee. His Majesty's Stationery Office: 1942; pp. 48 (iii) and 74 figures and 7 diagrams; with aids to the examination of the 
peripheral nervous system. By Michael O'Brien for the Guarantors of Brain. Saunders Elsevier: 2010; pp. [8] 64 and 94 Figures. Brain 20। 0 ; 133 ( I 0):2838-44. DOI: 10.1093/brain/awq270.

5. Ropper AH. Unusual clinical variants and signs in Guillain-Barré syndrome. Arch Neurol 1986;43(II):II50-2. DOI: 10.100I/ archneur. I 986.00520I I00440I 2.

6. Nagashima T, Koga M, Odaka M, Hirata K, Yuki N. Continuous spectrum of pharyngeal-cervicalbrachial variant of Guillain-Barré syndrome. Arch Neurol
2007;64(10):1519-23. DOI: 10 1001/archneur.64.10.1519.

7. Tariq M, Afreen A. Pharyngeal cervical brachial variant of GuillainBarré Syndrome requiring prolonged ventilatory support. Pak Armed Forces Med J 2017; 67(6): 1092-3.

\section{AUTHORS' CONTRIBUTIONS}

Following authors have made substantial contributions to the manuscript as under:

MF \& FA: Identified \& managed the case, manuscript witting, final approval of the version to be published

SBA: Diagnosis of the case, manuscript witting, final approval of the version to be published

Authors agree to be accountable for all aspects of the work in ensuring that questions related to the accuracy or integrity of any part of the work are appropriately investigated and resolved.

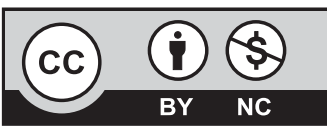

This is an Open Access article distributed under the terms of the Creative Commons Attribution-Non Commercial 2.0 Generic License.

KMUJ web address: www.kmuj.kmu.edu.pk

Email address: kmuj@kmu.edu.pk 